\title{
Dinâmica e estoque de carbono em floresta primária na região de Manaus/AM
}

\author{
Cintia Rodrigues de SOUZA ${ }^{1}$, Celso Paulo de AZEVEDO², Luiz Marcelo Brum ROSSI ${ }^{3}$, Kátia Emídio da \\ SILVA $^{4}$, Joaquim dos SANTOS 5 , Niro HIGUCHI ${ }^{6}$
}

\section{RESUMO}

Este trabalho analisou dados de três inventários florestais realizados na área da Floresta Experimental, pertencente à Embrapa Amazônia Ocidental e localizada no km 54 da BR-174, em Manaus/AM. O objetivo foi estudar a dinâmica da floresta - taxas de incremento, recrutamento e mortalidade - além do estoque de carbono, em uma área de floresta primária, sem qualquer tipo de intervenção. Os inventários foram realizados nos anos de 2005, 2007 e 2010, em 15 parcelas permanentes de 1 hectare cada, onde foram mensuradas todas as árvores com diâmetro a 1,3 m de altura do solo (DAP) superior a $10 \mathrm{~cm}$. Foram calculadas as taxas de recrutamento e mortalidade, os incrementos periódicos anuais (IPAs) em termos de DAP, área basal e volume, as biomassas fresca e seca acima do nível do solo e total e o estoque de carbono da vegetação. As taxas de recrutamento foram de $2 \%$ e $1,8 \%$ e as de mortalidade foram de $0,95 \%$ e $1,3 \%$ para os períodos entre 2005 e 2007 e 2007 e 2010 , respectivamente.

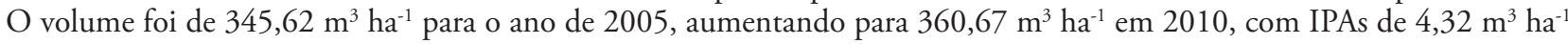
ano $^{-1}$ entre 2005 e 2007 e $1,31 \mathrm{~m}^{3}$ ha $^{-1}$ ano $^{-1}$ entre 2007 e 2010 . O estoque de carbono total teve um acréscimo de 173,63 t C ha- em 2005 para 181,01 t C ha ${ }^{-1}$ em 2010, confirmando que a floresta acumulou carbono no período, atuando assim como sumidouro.

PALAVRAS-CHAVE: recrutamento, mortalidade, incrementos, Amazônia, sequestro de carbono.

\section{Dynamics and carbon storage in primary forest in the region of Manaus /}

\section{AM}

\section{ABSTRACT}

This study analyzed data from three forest inventories conducted in the Experimental Forest, which belongs to Embrapa Western Amazon basin and is located at km 54 on BR-174, Manaus / AM. The aim was to study forest dynamics - the rates of recruitment, growth and mortality - in addition to carbon storage in an area of primary forest, without any intervention. The surveys were conducted in the years 2005, 2007 and 2010 in 15 permanent plots of 1 hectare each. There, all trees with diameter at breast height $(\mathrm{DBH})$ above $10 \mathrm{~cm}$ were measured. We calculated the rates of recruitment and mortality, the periodic annual increments (PAIs) in terms of $\mathrm{DBH}$, basal area and volume, fresh and dry biomass above the soil and total carbon storage in the vegetation. The recruitment rates were 2.0 percent and 1.8 percent, and mortality were 0.95 percent and 1.3 percent for the periods between 2005 and 2007 and 2007 and 2010 , respectively. The volume was $345.62 \mathrm{~m}^{3} \mathrm{ha}^{-1}$ for the year 2005, rising to $360.67 \mathrm{~m}^{3} \mathrm{ha}^{-1}$ in 2010 with PAIs of $4.32 \mathrm{~m}^{3} \mathrm{ha}^{-1} \mathrm{yr}^{-1}$ between 2005 and 2007 and $1.31 \mathrm{~m}^{3}$ ha $\mathrm{yr}^{-1}$ between 2007 and 2010. The total carbon storage increased from $173.63 \mathrm{t} \mathrm{C} \mathrm{ha}^{-1}$ in 2005 to $181.01 \mathrm{t} \mathrm{C} \mathrm{ha}^{-1}$ in 2010 , confirming that the forest accumulated carbon in the period, acting as a sink.

KEYWORDS: recruitment, mortality increments, Amazon, carbon sequestration.

1Embrapa Amazônia Ocidental e doutoranda INPA/CFT, cintia.souza@cpaa.embrapa.br

2Embrapa Amazônia Ocidental, celso.azevedo@cpaa.embrapa.br

3Embrapa Amazônia Ocidental, marcelo.rossi@cpaa.embrapa.br

[Embrapa Amazônia Ocidental, katia.emidio@cpaa.embrapa.br

5INPA, joca@inpa.gov.br

6INPA, niro@inpa.gov.br 


\section{INTRODUÇÃO}

Em discussôes sobre o futuro da floresta Amazônica, diversos segmentos da sociedade reconhecem a vocaçáo florestal desta região e consideram ser possível conciliar seu desenvolvimento com a conservação de seu imenso patrimônio natural (Azevedo 2006). Para o aproveitamento adequado dos recursos existentes, é necessário antes de tudo avaliar a dinâmica da floresta, procedendo ao seu monitoramento, por meio de inventário florestal contínuo (IFC), onde se efetuam mensuraçôes na floresta não perturbada em diferentes ocasióes, com o objetivo de avaliar seu comportamento frente às causas naturais de alteração (Azevedo 2006). Isso resulta na obtençâo de uma série de informaçôes sobre sua dinâmica, ou seja, sobre as mudanças ocorridas na estrutura da floresta, em sua composição, crescimento, mortalidade e regeneração, sobre fatores ecológicos que afetam essa dinâmica e a complexidade das interaçóes entre os fatores envolvidos no processo (Silva 1989). Tais informaçôes são essenciais para os planejadores do manejo florestal, pois auxiliaráo no momento de tomada de decisóes com respeito ao ciclo, diâmetro mínimo e volume de corte, entre outros (Azevedo 2006).

Considerando a expressiva cobertura vegetal da região amazônica, cada vez mais se tem evidenciado sua importância no controle das mudanças climáticas globais, tanto pela capacidade de emitir gases do efeito estufa para a atmosfera, via queimadas ou desmatamentos, como de absorver carbono da atmosfera por meio do crescimento do povoamento.

Diversos estudos avaliam se as florestas tropicais náo manejadas são consideradas fonte ou sumidouro de carbono. Por um lado, a floresta pode agir como sumidouro, pois as árvores absorvem carbono durante a fotossíntese e estocam o excesso na forma de biomassa (Malhi et al. 1998; Phillips et al. 1998; Nobre 2001; Higuchi et al. 2004). Porém, as florestas também podem ser fonte de carbono, devido à diminuição da produtividade florestal e aumento nas taxas de mortalidade decorrentes do aumento da temperatura e da severidade das secas, principalmente causadas pelo fenômeno El Niño (Clark 2004), ou devido ao desmatamento, fonte significativa de gases de efeito estufa para a atmosfera (Fearnside 1997; Houghton et al. 2000).

Estima-se que o estoque de carbono florestal na Amazônia brasileira, incluindo a necromassa e a biomassa abaixo no solo, seja de aproximadamente 86 bilhóes de toneladas (Saatchi et al. 2007). Atualmente, o desmatamento da Amazônia representa $75 \%$ das emissóes brasileiras e 20\% das emissōes globais de gases de efeito estufa (Houghton 2003; MCT 2010).

$\mathrm{Na}$ área florestal, existem quatro opçóes de mitigaçáo dos efeitos das mudanças climáticas globais: manejo florestal, redução do desmatamento, florestamento e reflorestamento. Segundo o $4^{\circ}$ Relatório de Avaliação do IPCC (Painel Inter- governamental sobre Mudanças do Clima), no curto prazo, os benefícios da mitigação por meio do desmatamento evitado (ou seja, conservaçấo das florestas) são maiores do que os benefícios do reflorestamento e do florestamento (IPCC 2007).

O objetivo deste trabalho foi estudar a dinâmica da vegetação arbórea, no que diz respeito às taxas de recrutamento, incremento e mortalidade, além do estoque de carbono de uma floresta tropical úmida não explorada na região de Manaus/AM, a fim de determinar se a floresta em questáo é um sumidouro ou uma fonte de carbono para a atmosfera.

\section{MATERIAL E MÉTODOS}

\section{Localização e caracterização da área de estudo}

Este trabalho está inserido no âmbito do Projeto Manejo Florestal na Amazônia, financiado com recursos da Embrapa. O estudo foi realizado na área denominada Floresta Experimental, localizada na Estação Experimental do Distrito Agropecuário da Suframa, pertencente à Embrapa Amazônia Ocidental, no km 54 da Rodovia BR-174, no município de Manaus/AM. A área fica compreendida entre as coordenadas

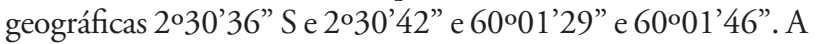
Floresta Experimental compreende uma área de 400 hectares de floresta primária, demarcada especificamente para estudos florestais e ecológicos, por não ter sofrido alteraçôes ambientais nem efeito de borda.

A cobertura florestal é típica da floresta tropical úmida densa de terra-firme. O clima é do tipo Am, classificaçáo de Köeppen, quente e úmido, com precipitação média anual entre 1.355 e $2.839 \mathrm{~mm}$. A temperatura média anual varia de 25,6 a $27,6{ }^{\circ} \mathrm{C}$ e a umidade relativa do ar é de $84 \%$ a $90 \%$, em média. A altitude varia entre 50 e $100 \mathrm{~m}$ e os solos predominantes são os Latossolos Amarelos distróficos com textura argilosa (Silva et al. 2008).

Silva et al. (2011) identificaram, nas mesmas parcelas estudadas neste trabalho, 8.771 indivíduos com diâmetro superior a $10 \mathrm{~cm}$, distribuídos em 264 espécies e 53 famílias. Grande parte dos indivíduos concentra-se em poucas espécies, tais como Protium hebetatum Daly, Eschweilera coriacea (DC.) S.A. Mori e Licania oblongifolia Standl., que somaram $21 \%$ do total. Segundo o estudo, as famílias Sapotaceae, Lecythidaceae e Burseraceae somaram 39\% do número total de indivíduos.

\section{Descrição do experimento}

A área da Floresta Experimental está dividida em 400 parcelas de 1,0 hectare cada (100 m x $100 \mathrm{~m}$ ). Quinze destas parcelas, previamente selecionadas de forma aleatória, foram divididas em 100 subparcelas de $100 \mathrm{~m}^{2}(10 \times 10 \mathrm{~m})$. Nos meses de abril de 2005, novembro de 2007 e novembro de 
2010 foram realizados inventários florestais nestas 15 parcelas permanentes, mensurando-se $100 \%$ das árvores com diâmetro a 1,3 m de altura do solo (DAP) superior a $10 \mathrm{~cm}$, a fim de avaliar as taxas de incremento, mortalidade e recrutamento da floresta. Nestes inventários foram avaliados os seguintes parâmetros para cada árvore: 1) identificaçáo botânica das espécies; 2) DAP; 3) classe de identificação do fuste (CIF); 4) presença de danos/defeitos na árvore; 5) presença ou náo de podridão; 6) grau de iluminação da copa; 6) presença de cipós e 7) coordenadas cartesianas. Todos os indivíduos mensurados foram plaqueteados e identificados botanicamente em nível de espécie. $\mathrm{O}$ material botânico foi identificado por meio de comparaçóes a exsicatas disponíveis no herbário do Instituto Nacional de Pesquisas da Amazônia (INPA).

Os DAPs foram medidos utilizando-se fita diamétrica, para o caso de árvores com fuste sem deformidades ou sapopemas. Quando o ponto de medição apresentou qualquer tipo de irregularidade ou sapopemas, a medição foi feita em um local imediatamente acima, desde que seja livre dessas anormalidades (Phillips et al. 1998).

A identificação das espécies foi feita por meio do nome científico, a fim de assegurar maior confiabilidade em todas as análises e possibilitar comparaçóes com as amostras do herbário do INPA. Quando houve algum indivíduo pertencente à espécie não identificada nos levantamentos anteriores, foi coletado material botânico (folhas, flores e frutos, preferencialmente) para posterior identificação.

\section{Dinâmica da vegetação arbórea}

Com os dados coletados, foram determinados os seguintes parâmetros referentes à dinâmica da floresta:

- Taxas de recrutamento e mortalidade, por meio das seguintes equaçóes:

$$
\begin{aligned}
& R \%=\frac{\text { núm. árv. ingressaram }}{\text { núm. árv. total da medição anterior }} \times 100 \\
& M \%=\frac{\text { núm. árv. morreram }}{\text { núm. árv. total da medição anterior }} \times 100
\end{aligned}
$$

Em que:

$\mathrm{R} \%$ = taxa de recrutamento;

$\mathrm{M} \%$ = taxa de mortalidade.

- Incremento periódico anual (IPA), em termos de DAP, área basal e volume:

$$
I P A=\frac{M_{2}-M_{1}}{A_{2}-A_{1}}
$$

Em que:

$\mathrm{M}_{2}$ e $\mathrm{M}_{1=}$ medidas nos tempos 2 e 1 , respectivamente; $A_{2}$ e $A_{1}=\operatorname{anos} 2$ e 1 , respectivamente
Para o cálculo dos IPAs, no caso do primeiro intervalo entre os inventários florestais realizados (de abril de 2005 a novembro de 2007), foi considerado o intervalo de 31 meses, o que corresponde a 2,6 anos. Para o segundo intervalo entre as mediçóes (de novembro de 2007 a novembro de 2010), foi considerado o intervalo exato de 36 meses ou 3 anos.

O volume foi obtido pelo emprego da equação apresentada por Higuchi et al. (1997) para a área em estudo:

$$
\begin{aligned}
& \mathrm{LnV}=-7,335+2,121 \mathrm{Ln}(\mathrm{DAP}) \\
& \mathrm{R}^{2}=0,95 \\
& \text { Em que: } \\
& \mathrm{Ln}=\text { logaritmo neperiano; } \\
& \mathrm{V}=\text { volume comercial com casca } \mathrm{em} \mathrm{m}^{3} .
\end{aligned}
$$

\section{Biomassa/carbono}

Para determinação dos teores de biomassa e carbono, foram utilizadas as equaçóes propostas por Silva (2007).

$$
\begin{aligned}
& \mathrm{PF}_{\text {abg }}=2,2737 \times \mathrm{DAP}^{1,9156} \\
& \mathrm{R}^{2}=0,85 \\
& \mathrm{PF}_{\text {tot }}=2,7179 \times \mathrm{DAP}^{1,8774} \\
& \mathrm{R}^{2}=0,94 \\
& \mathrm{PS}_{\text {abg }}=\left(\mathrm{PF}_{\text {abg }}\right) \times 0,592 \\
& \mathrm{PS}_{\text {tot }}=\left(\mathrm{PF}_{\text {tot }}\right) \times 0,584 \\
& \mathrm{C}_{\text {abg }}=\left(\mathrm{PS}_{\text {abg }}\right) \times 0,485 \\
& \mathrm{C}_{\text {tot }}=\left(\mathrm{PS}_{\text {tot }}\right) \times 0,485
\end{aligned}
$$

Em que:

$\mathrm{PF}_{\text {abg }}=$ biomassa fresca acima do nível do solo;

$\mathrm{P}_{\text {tot }}=$ biomassa fresca total (acima do nível do solo + raízes grossas);

$\mathrm{PS}_{\mathrm{abg}}=$ biomassa seca acima do nível do solo;

$\mathrm{PS}_{\text {tot }}=$ biomassa seca total;

$\mathrm{C}_{\mathrm{abg}}=$ carbono da vegetação acima do nível do solo;

$\mathrm{C}_{\text {tot }}=$ carbono total acima do nível do solo + raízes grossas).

Os dados de incrementos (em DAP, área basal, volume e carbono) foram analisados utilizando-se o Teste-t pareado (amostras dependentes), por meio do software estatístico Systat $12.0^{\circ}$.

\section{RESULTADOS E DISCUSSÃO}

Os valores médios de recrutamento e mortalidade (em número de indivíduos por hectare por ano e em porcentagem) para a área avaliada são apresentados na Tabela 1 .

Tabela 1 -Valores médios de recrutamento e mortalidade, em número de indivíduos por hectare por ano e em porcentagem, para os anos de 2007 e 2010 na área da Floresta Experimental da Embrapa Amazônia Ocidental.

\begin{tabular}{lcccc}
\hline & 2007 & \multicolumn{2}{c}{2010} \\
\hline & No ind. ha-1 $^{-1}$ ano $^{-1}$ & $\%$ & No ind. ha $^{-1}$ ano $^{-1}$ & $\%$ \\
Recrutamento & 12,1 & 2,0 & 10,6 & 1,8 \\
Mortalidade & 5,7 & 0,95 & 7,7 & 1,3 \\
\hline
\end{tabular}


Pela Tabela 1 pode-se perceber que, quando se leva em consideração os valores médios, a floresta estudada teve mais indivíduos sendo recrutados (o que representa acréscimo de árvores no povoamento) do que indivíduos mortos (por causas naturais). Isso náo aconteceu apenas em uma parcela (em ambos os anos de avaliação) e em três parcelas no ano de 2010, quando as taxas de mortalidade superaram as de recrutamento.

Diversos autores encontraram taxas de mortalidade inferiores às registradas no presente estudo. Em trabalho realizado por Colpini et al. (2010), a taxa de mortalidade de uma floresta em Mato Grosso foi de 0,78\%. Higuchi et al. (2004) encontraram valor de $0,7 \%$ de mortalidade para uma floresta na regiáo de Manaus (AM), entre 1986 e 2000.

No trabalho realizado por Higuchi et al. (2004) em uma floresta primária na mesma região, os autores também obtiveram resultado menor ao da área deste estudo, com 0,7\% de recrutamento para o período de 1986 a 2000.

A Tabela 2 contém os valores médios de número de indivíduos, área basal por hectare, volume por hectare, biomassa fresca total e carbono total (acima do nível do solo + raízes grossas), para os anos de avaliação. Verifica-se que, para todos os parâmetros considerados, seus respectivos valores sempre apresentaram crescimento de um ano para outro, confirmando que a floresta acumulou biomassa durante o período avaliado. É possível também chegar a essa mesma conclusão analisando-se a Tabela 3, na qual constam os incrementos periódicos anuais (IPAs) para estes parâmetros. Os valores dos incrementos periódicos anuais em DAP, área basal, volume e estoque de carbono diferiram estatisticamente entre si nos dois períodos analisados (de 2005 a 2007 e de 2007 a 2010), indicando que provavelmente a floresta estudada não esteve em equilíbrio neste período e acumulou carbono de forma mais significativa do que emitiu pela mortalidade das árvores.

Para a Amazônia, diversos estudos encontraram valores de biomassa fresca acima do nível do solo. Utilizando dados de 216 parcelas permanentes instaladas em áreas de terrafirme na região amazônica, Saatchi et al. (2007) estimaram a

Tabela 2 - Valores médios (média \pm desvio padrão), para os 15 ha estudados, de área basal $(A B)$, volume $(\mathrm{V})$, biomassa fresca total (Ptotal) e estoque de carbono total (Ctotal) para os anos de 2005, 2007 e 2010 (considerando os indivíduos com DAP $\geq 10 \mathrm{~cm}$ ).

\begin{tabular}{|c|c|c|c|c|c|}
\hline Ano & $\begin{array}{l}\text { Número de } \\
\text { indivíduos }\end{array}$ & $\begin{array}{l}A B \\
\left(m^{2} h a^{-1}\right)\end{array}$ & $\begin{array}{l}V \\
\left(m^{3} h a^{-1}\right)\end{array}$ & $\begin{array}{l}P_{\text {total }} \\
\left(\mathrm{t}^{-1} \mathrm{a}^{-1}\right)\end{array}$ & $\begin{array}{l}\mathrm{C}_{\text {total }} \\
\left.\text { (t ha- }{ }^{-1}\right)\end{array}$ \\
\hline 2005 & $\begin{array}{l}578,33 \pm \\
65,6\end{array}$ & $\begin{array}{l}27,16 \pm \\
2,70\end{array}$ & $\begin{array}{l}345,62 \pm \\
38,01\end{array}$ & $\begin{array}{l}613,01 \pm \\
55,84\end{array}$ & $\begin{array}{l}173,63 \pm \\
15,82\end{array}$ \\
\hline 2007 & $\begin{array}{l}595,00 \pm \\
68,5\end{array}$ & $\begin{array}{l}28,01 \pm \\
2,96\end{array}$ & $\begin{array}{l}356,77 \pm \\
41,35\end{array}$ & $\begin{array}{l}631,90 \pm \\
61,28\end{array}$ & $\begin{array}{l}178,98 \pm \\
17,36\end{array}$ \\
\hline 2010 & $\begin{array}{l}603,73 \pm \\
64,9\end{array}$ & $\begin{array}{l}28,33 \pm \\
2,68\end{array}$ & $\begin{array}{l}360,67 \pm \\
37,27\end{array}$ & $\begin{array}{l}639,05 \pm \\
55,75\end{array}$ & $\begin{array}{l}181,01 \pm \\
15,79\end{array}$ \\
\hline
\end{tabular}

biomassa média destas florestas em 254,8 $\mathrm{t} \mathrm{ha}^{-1}$. Já Fearnside $(1997,2000)$ estimou a biomassa total na Amazônia brasileira (considerando-se a biomassa acima e abaixo do solo, inclusive a necromassa, mas sem considerar o carbono no solo) em valores entre 433,6 e $464 \mathrm{t} \mathrm{ha}^{-1}$ em média, considerando as florestas densas e não densas.

Houghton et al. (2000), utilizando dados do projeto RADAMBRASIL, fizeram estimativas de biomassa para a Amazônia brasileira que variaram de 66 a 277 t C ha ${ }^{-1}$. Os autores consideraram que $20 \%$ deste valor se referem à biomassa abaixo do solo e que $50 \%$ da biomassa é composta por carbono. Já Houghton et al. (2001) estimaram biomassa média de florestas de terra-firme em $177 \mathrm{t} \mathrm{C} \mathrm{ha-1}$.

Estudos de Higuchi et al. (1998) apresentaram valores de volume superiores ao do presente estudo. Em dois transectos na região da bacia do Rio Cuieiras (Manaus/AM), o volume médio encontrado para a floresta não manejada foi de $430,5 \mathrm{~m}^{3} \mathrm{ha}^{-1}$. Nesta mesma área de estudo, Teixeira et al. (2007) encontraram valores inferiores, de $323,97 \mathrm{~m}^{3}$ ha $^{-1}$ para o ano 2000 e $346,38 \mathrm{~m}^{3} \mathrm{ha}^{-1}$ para o ano de 2004.

Tabela 3 - Incrementos periódicos anuais (IPA) em DAP, área basal (AB), volume (V) e estoque de carbono (C) encontrados no período estudado para 0 total dos indivíduos na Floresta Experimental da Embrapa Amazônia Ocidental.

\begin{tabular}{|c|c|c|}
\hline Variáveis & IPA 2007-2005 & IPA 2010-2007 \\
\hline $\operatorname{DAP}\left(\mathrm{cm} \mathrm{ano} 0^{-1}\right)^{* *}$ & 0,21 & 0,13 \\
\hline$A B\left(m^{2}\right.$ ha $^{-1}$ ano $\left.^{-1}\right) * *$ & 0,33 & 0,12 \\
\hline$V\left(m^{3}\right.$ ha $^{-1}$ ano $\left.{ }^{-1}\right) * *$ & 4,32 & 1,31 \\
\hline $\mathrm{C}\left(\mathrm{t} \mathrm{ha}^{-1} \mathrm{ano}^{-1}\right)^{*}$ & 2,06 & 0,67 \\
\hline
\end{tabular}

* Diferença estatística significativa entre os incrementos periódicos anuais em carbono nos dois períodos analisados $(p=0,003)$.

** Diferença estatística significativa entre os incrementos periódicos anuais em DAP, área basal e volume nos dois períodos analisados $(p=0,000)$.

Oliveira e Braz (2006), estudando uma área de floresta manejada em Pedro Peixoto (AC), encontraram maiores valores de incremento em diâmetro, como esperado em relação a uma área não manejada. Os valores variaram de $2 \mathrm{~cm}$ ano-1, para espécies pioneiras, a $0,1 \mathrm{~cm}$ ano ${ }^{-1}$, para algumas espécies de sub-bosque.

Os resultados dos incrementos periódicos anuais (IPA) em área basal para a área estudada são superiores ao encontrado por Oliveira et al. (2005), que trabalhando na Floresta Nacional do Tapajós, em Belterra (PA), encontraram incremento de 0,06 $\mathrm{m}^{2}$ ha- $^{-1}$ ano- ${ }^{1}$ para uma área sem intervenção. Teixeira et al. (2007), estudando floresta na região de Manaus, encontraram incremento em área basal superior: $0,44 \mathrm{~m}^{2} \mathrm{ha}^{-1}$ ano- ${ }^{-1}$.

Para uma floresta sem intervençấo no Acre, Oliveira et al. (2005) apresentaram resultado de incremento periódico anual em volume inferior ao apresentado neste trabalho, de 0,70 $\mathrm{m}^{3} \mathrm{ha}^{-1}$ ano $^{-1}$. Na região de Santarém (PA), Silva et al. (1996) observaram o crescimento e a produçáo numa floresta de 
Tabela 4 - Estoques e incrementos de carbono para as famílias mais representativas da área estudada.

\begin{tabular}{llllll}
\hline Família & $\begin{array}{l}\text { Estoque C em 2005 } \\
\left(\mathrm{t} \mathrm{ha}^{-1}\right)\end{array}$ & $\begin{array}{l}\text { Estoque C em 2007 } \\
\left(\mathrm{t} \mathrm{ha}^{-1}\right)\end{array}$ & $\begin{array}{l}\text { Estoque C em 2010 } \\
\left(\mathrm{t} \mathrm{ha}^{-1}\right)\end{array}$ & $\begin{array}{l}\text { Incremento C }(\mathrm{t} \mathrm{ha-1}) \\
2010-2007\end{array}$ & $\begin{array}{l}\text { Incremento C }(\mathrm{t} \text { ha-1 } \\
2007-2005\end{array}$ \\
\hline Fabaceae & 354,87 & 370,12 & 439,74 & 69,62 & 15,25 \\
\hline Sapotaceae & 262,30 & 268,43 & 344,19 & 75,76 & 6,14 \\
Lecythidaceae & 242,11 & 246,30 & 317,62 & 71,32 & 4,19 \\
Burseraceae & 194,83 & 202,30 & 207,46 & 5,16 & 7,46 \\
Chrysobalanaceae & 168,04 & 169,68 & 180,55 & 10,86 & 1,64 \\
Moraceae & 119,55 & 120,92 & 145,62 & 24,70 & 1,37 \\
Humiriaceae & 105,33 & 108,53 & 109,80 & 1,27 & 3,19 \\
Lauraceae & 95,03 & 99,01 & 107,59 & 8,58 & 3,98 \\
\hline
\end{tabular}

terra-firme não explorada, sendo que o incremento periódico anual em volume foi de $1,6 \mathrm{~m} 3 \mathrm{ha}^{-1}$ ano- $^{1}$.

Clark (2004) afirma que estudos apontam que as florestas tropicais atuam como importante reservatório de carbono, possivelmente em resposta ao aumento na concentração de carbono na atmosfera, que aumenta a produtividade da floresta. Estudos baseados em inventários florestais e baseados no cálculo de inversão dos transportes atmosféricos de $\mathrm{CO}_{2}$ também colocam a Amazônia como sumidouro de parte do excesso de carbono na atmosfera, com totais entre 0,2 e 0,5 Gt C ano ${ }^{-1}$, o que significa que a Amazônia como um todo poderia responder por 10 a $20 \%$ do sumidouro global de carbono da biota terrestre (Nobre 2001).

Higuchi et al. (2004), estudando uma floresta primária na regiāo de Manaus (AM), concluíram que a floresta sequestra carbono a uma taxa de 1,2 $\mathrm{tha}^{-1} \mathrm{ano}^{-1}$, devido principalmente ao crescimento individual das árvores armazenadas dentro do sistema, compensando as perdas causadas pela mortalidade natural.

Conforme a Tabela 4, as famílias que mais contribuíram para este estoque foram basicamente as mesmas nos três anos de estudo, sendo, em ordem decrescente: Fabaceae, Sapotaceae, Lecythidaceae, Burseraceae, Chrysobalanaceae, Moraceae, Humiriaceae e Lauraceae .

\section{CONCLUSÕES}

A floresta estudada apresentou, no período entre 2005 e 2010, acréscimo de indivíduos superiores a $10 \mathrm{~cm}$ de DAP (recrutamento maior que a mortalidade), sendo que estes indivíduos apresentaram incremento em área basal, volume, biomassa fresca total e estoque de carbono. Assim, no período estudado a floresta primária náo apresentou equilíbrio, tendo atuado como sumidouro de carbono da atmosfera.

\section{AGRADECIMENTOS}

À Vilany M. C. Carneiro, pelo auxílio com a identificação botânica das espécies.

\section{BIBLIOGRAFIA CITADA}

Azevedo, C.P. 2006. Dinâmica de florestas submetidas a manejo na Amazônia Oriental: experimentaçâo e simulação. Tese (Doutorado em Engenharia Florestal) - Universidade Federal do Paraná, Curitiba/PR. 254 pp.

Clark, D. A. 2004. Sources or sinks? The responses of tropical forests to current and future climate and atmospheric composition. Philosophical Transactions of the Royal Society of London B, 359: 477-491.

Colpini, C.; Silva, V. S. M.; Soares, T. S.; et al. 2010. Incremento, ingresso e mortalidade em uma floresta de contato ombrófila aberta/estacional em Marcelândia, Mato Grosso. Acta Amazonica, 40 (3): 549-556.

Fearnside, P. M. 1997. Greenhouse gases from deforestation in Brazilian Amazonia: net committed emissions. Climatic Change, 35(3): 321-360.

Fearnside, P. M. 2000. Global warming and tropical land-use change: greenhouse gás emissions from biomass burning, decomposition and soils in forest conversion, shifting cultivation and secondary vegetation. Climatic Change, 46 (1-2): 115-158.

Higuchi, N.; Santos, J.; Ribeiro, J. R.; Freitas, J. V. de; Vieira, G.; Cöic, A.; Minette, L. 1997. Crescimento e Incremento de uma Floresta Amazônica de Terra-firme Manejada Experimentalmente, p. 89-131. In: Biomassa e Nutrientes Florestais - Relatório Final do Projeto Bionte. MCT-INPA. Manaus.

Higuchi, N.; Santos, J.; Ribeiro, R.J.; Minette, L.; Biot, Y. 1998. Biomassa da parte aérea da vegetaçáo da floresta tropical úmida de terra firme da Amazônia brasileira. Acta Amazonica, 28 (2): 153-166.

Higuchi, N.; Chambers, J.; Santos, J.; Ribeiro, R. J.; Pinto, A. C. M.; Silva, R. P. da; Rocha, R. de M.; Tribuzy, E. S. 2004. Dinâmica e balanço do carbono da vegetação primária da Amazônia Central. Revista Floresta, 34 (3): 295-304.

Houghton, R. A.; Skole, D. L.; Nobre, C. A.; Hackler, J. L.; Lawrence, K. T.; Chomentowski, W. H. 2000. Annual fluxes of carbon from deforestation and regrowth in the Brazilian Amazon. Nature, 403: 301-304.

Houghton, R. A.; Lawrence, K. T.; Hackler, J. L.; Brown S. 2001. The spatial distribution of forest biomass in the Brazilian Amazon: a comparison of estimates. Global Change Biology, 7: 731-746. 
Houghton, R. A. 2003. Revised estimates of the annual net flux of carbon to the atmosphere from changes in land use and land management 1850-2000. Tellus 55B: 378-390.

IPCC. 2007. Fourth Assessment Report of the Intergovernmental Panel on Climate Change. [B. Metz, O.R. Davidson, P.R. Bosch, R. Dave, L.A. Meyer (eds)], Cambridge University Press, Cambridge, United Kingdom and New York, NY, USA.

Malhi, Y.; Nobre, A. D., Grace, J.; Kruijt B.; Pereira M. G. P.; Culf A.; Scott S.1998. Carbon dioxide transfer over a Central Amazonian rain forest. Journal of Geophysical Research-Atmospheres, 103: 31593-31612.

MCT. 2010. Segunda Comunicação Nacional do Brasil à ConvençãoQuadro das Nações Unidas sobre Mudança Climática. Brasília/ DF. 520 pp.

Nobre, C. A. 2001. Amazônia: fonte ou sumidouro de carbono? p. 197-224. In: MMA. Causas e dinâmica do desmatamento na Amazônia, Brasília, DF.

Oliveira, M. V. N.; Braz, E. M. 2006. Estudo da dinâmica da floresta manejada no projeto de manejo florestal comunitário do PC Pedro Peixoto na Amazônia Ocidental. Acta Amazonica, 36 (2): 177-182.

Oliveira, L. C.; Couto, H. T. Z.; Silva, J. N. M.; Carvalho, J. O. P. 2005. Efeito da exploraçáo de madeira e tratamentos silviculturais na composição florística e diversidade de espécies em uma área de 136 ha na Floresta Nacional do Tapajós, Belterra, Pará. Scientia Forestalis, 69: 62-76.

Phillips, O. L.; Malhi, Y.; Higuchi, N.; Laurance, W. F.; Núñez, P. V.; Vásquez, R. M.; Laurance, S. G.; Ferreira, L. V.; Stern, M.; Brown, S.; Grace, J. 1998. Changes in the carbon balance of tropical forests: evidence from long-term plots. Science, 282:439-442.

Saatchi, S. S.; Houghton, R. A.; Alvalá, R. C. S.; Soares, J. V.; Yu, Y. 2007. Distribution of aboveground live biomass in the Amazon basin. Global Change Biology, 13: 816-837.

Silva, J. N. M. 1989. The behavior of the tropical rain forest of the Brazilian Amazon after logging. Thesis (PhD) - University of Oxford, Oxford, United Kingdom, 303 pp.

Silva, J. N. M.; Carvalho, J. O. P.; Lopes, J. C. A.; Oliveira, R. P., Oliveira, L. C. 1996. Growth and yield studies in the Tapajós region, Central Brazilian Amazon. Commonwealth Forestry Review, 75 (4), 325-329. 1996.

Silva, K. E.; Matos, F. D. A.; Ferreira, M. M. 2008. Composição florística e fitossociologia de espécies arbóreas do Parque Fenológico da Embrapa Amazônia Ocidental. Acta Amazonica, 38 (2):213-222.

Silva, K. E. S.; Martins, S. V.; Ribeiro, C. A. A. S.; Santos, N. T.; Azevedo, C. P.; Matos, F. D. A.; Amaral, I. L. 2011. Floristic composition and similarity of 15 hectares in Central Amazon, Brazil. Revista de Biología Tropical, 59 (4): 1927-1938.

Silva, R. P. 2007. Alometria, estoque e dinâmica da biomassa de florestas primárias e secundárias na região de Manaus/AM. Tese (Doutorado) - Instituto Nacional de Pesquisas da Amazônia (INPA/UFAM), Manaus/AM. 152 pp.

Teixeira, L. M.; Chambers, J. Q.; Silva, A. R.; Lima, A. J. N.; Carneiro, V. M. C.; Santos, J.; Higuchi, N. Lima, A. J. N.; Carneiro, V. M. C.; Santos, J.; Higuchi, N. . 2007. Dynamics of the Terra-firme primary forest in Manaus-AM region using the Markov probabilistic transition matrix. Acta Amazonica, 37 (3): 377-384. (in Portuguese, with abstract in English).

Recebido em: 14/09/2011

Aceito em: 26/12/2011 